\title{
Deformable Registration of Multi-Modal Data Including Rigid Structures
}

\author{
Ronald H. Huesman, Senior Member, IEEE, Gregory J. Klein, Member, IEEE, \\ Joey A. Kimdon, Student Member, IEEE, Chaincy Kuo, Sharmila Majumdar
}

\begin{abstract}
Multi-modality imaging studies are becoming more widely utilized in the analysis of medical data. Anatomical data from CT and MRI are useful for analyzing or further processing functional data from techniques such as PET and SPECT. When data are not acquired simultaneously, even when these data are acquired on a dual-imaging device using the same bed, motion can occur that requires registration between the reconstructed image volumes. As the human torso can allow non-rigid motion, this type of motion should be estimated and corrected.

We report a deformation registration technique that utilizes rigid registration for bony structures, while allowing elastic transformation of soft tissue to more accurately register the entire image volume. The technique is applied to the registration of CT and MR images of the lumbar spine. First a global rigid registration is performed to approximately align features. Bony structures are then segmented from the CT data using a semi-automated process, and bounding boxes for each vertebra are established. Each CT subvolume is then individually registered to the MRI data using a piece-wise rigid registration algorithm and a mutual information image similarity measure. The resulting set of rigid transformations allows for accurate registration of the parts of the CT and MRI data representing the vertebrae, but not the adjacent soft tissue. To align the soft tissue, a smoothly-varying deformation is computed using a thin plate spline (TPS) algorithm. The TPS technique requires a sparse set of landmarks that are to be brought into correspondence. These landmarks are automatically obtained from the segmented data using simple edge-detection techniques and random sampling from the edge candidates. A smoothness parameter is also included in the TPS formulation for characterization of the stiffness of the soft tissue. Estimation of an appropriate stiffness factor is obtained iteratively by using the mutual information cost function on the result of the global deformable transformation.
\end{abstract}

Keywords - multi-modality, registration, lumbar spine, rigid, elastic, X-ray CT, MRI

\section{INTRODUCTION}

$\mathrm{M}$ ULTI-MODALITY imaging studies are becoming more widely utilized in the analysis of medical data.

This work was supported by the National Institute on Aging, U.S. Department of Health and Human Services under grant R01AG17762, by the Director, Office of Science, Office of Biological and Environmental Research, Medical Sciences Division of the U.S. Department of Energy under contract DE-AC03-76SF00098, and by a National Science Foundation Graduate Research Fellowship.

R. H. Huesman is with the Center for Functional Imaging, Lawrence Berkeley National Laboratory, Berkeley, CA 94720 USA. E-mail RHHuesman@lbl.gov

G. J. Klein and C. Kuo were also with the Lawrence Berkeley National Laboratory. G. J. Klein is currently with QuantifiCare, F06902 Sophia Antipolis, France. C. Kuo is currently with Xenogen Corporation, Alameda, CA 94501 USA.

J. A. Kimdon is with the Electrical Engineering and Computer Sciences Department, University of California, Berkeley, CA 94720 USA. E-mail jkimdon@eecs.berkeley.edu

S. Majumdar is with the Magnetic Resonance Science Center, University of California, San Francisco, CA 94143 USA.
X-ray computed tomography (CT) data depict high contrast structure of bony tissue, magnetic resonance imaging (MRI) provides excellent contrast of soft tissue, and nuclear imaging techniques such as positron emission tomography (PET) and single photon emission computed tomography (SPECT) provide functional data. Analysis of the data is enhanced by the complementary information present in a multi-modality study, and techniques exist that use anatomical datasets to further process functional images. In order to analyze or utilize data from two different modalities, accurate registration is necessary.

Most registration algorithms used in medical imaging assume that objects captured in the images move as a single rigid body. This may be appropriate for imaging of the head, but for studies of the human torso, a more sophisticated motion description is desired. Soft tissue within the torso can deform elastically, yet there are also structures, usually bone, that are constrained to move locally in a rigid fashion.

This work is part of a study investigating the progression of degenerative diseases in the spine and knee, in particular spinal stenosis and osteoarthritis. Such diseases can affect both bone and soft tissue, so the accurate registration of both rigid and non-rigid structures is important. We report here a registration technique that permits rigid alignment of bony structures while allowing elastic transformation of soft tissue to more accurately register the entire image volume.

In this paper, the registration technique is applied to CT and MR spine datasets. Past authors have attempted to register multi-modal datasets of the torso or spine using landmarks and globally rigid techniques [1]. A globally elastic technique has been proposed for registration of PET and CT torso data [2], and a deformation technique incorporating rigid structures has also been reported which uses manually specified landmarks and segmented rigid structures [3]. The current work makes use of automatically defined landmarks in a hybrid rigid/non-rigid registration of volumetric data. Separate rigid transformations are used to describe the motion of each segmented bony structure, and the motion of soft tissue is described by a thin plate spline (TPS). The TPS has been implemented to model elastic stiffness matching the actual image data.

\section{Methods}

Image datasets of the lumbar spine were acquired using a GE 1.5T Signa MRI scanner and a GE multi-detector CT scanner. Axial T1 MRI images were acquired using spin 
echo imaging with $\mathrm{TR}=633 \mathrm{msec}, \mathrm{TE}=9 \mathrm{msec} .256 \times 256$ x 42 voxel MRI volumes were acquired from a volunteer using a voxel size of $0.7 \mathrm{~mm} \times 0.7 \mathrm{~mm}$ x $3.0 \mathrm{~mm}$. CT data were acquired to produce a $512 \times 512 \times 75$ voxel volume with $0.35 \mathrm{~mm} \times 0.35 \mathrm{~mm} \times 3.0 \mathrm{~mm}$ voxels. To produce smaller datasets with finer axial sampling, the CT data were resampled into $128 \times 128 \times 107$ voxel volumes with voxel size $1.4 \mathrm{~mm}$ x $1.4 \mathrm{~mm}$ x $2.1 \mathrm{~mm}$ using trilinear interpolation. In addition, to test our algorithm on data sets with a larger range of motion, we obtained images of the isolated lumbar spine of a cadaver, which was fitted from a previous study into a frame to allow intentional flexion and extension. We used $256 \times 256 \times 9$ voxel MRI volumes (1.0 mm x $1.0 \mathrm{~mm} \times 5.0 \mathrm{~mm}$ ) from this previous study and acquired corresponding 512 × 512 x 109 voxel CT datasets at $0.35 \mathrm{~mm} \times 0.35 \mathrm{~mm} \times 1.25 \mathrm{~mm}$, in neutral, extended, and flexed positions.

The goal of the registration process is to correctly and automatically deform the MRI volume so that it matches the reference CT volume. The first step in this task is the segmentation of the vertebrae from the background in the CT data. This was carried out using a semi-automatic process using a sequence of thresholding, 3D region growing and filtering operations combined with manual editing.

Registration of the CT to the MRI dataset requires an appropriate metric to compare how well the two image volumes are aligned. The mutual information cost function rewards alignments that group together similar structures, even if the intensity levels in the two images are not directly correlated, as is the case with different imaging modalities. This quality has made the measure useful in registering multi-modal datasets [4]-[6]. Mutual information $(M I)$ is defined in terms of the entropies of two images, $A$ and $B$, as follows:

$$
M I(A, B)=H(A)+H(B)-H(A, B),
$$

where $H(A)$ and $H(B)$ represent the entropies of $A$ and $B$, and $\mathrm{H}(\mathrm{A}, \mathrm{B})$ is the joint entropy of the two images. Entropy is defined as:

$$
H(A)=-\sum_{a} p_{A}(a) \log p_{A}(a)
$$

where $p_{A}(a)$ is the probability distribution of intensity values of the image A. In this paper, as has been done in the past, the probability distributions are approximated using a histogram of the image voxel values.

We use this metric first in a globally rigid registration scheme to approximately align features in the MRI dataset with those in the $\mathrm{CT}$. Global rotation and translation parameters are optimized using Powell's method. Once the global registration has been obtained, the hybrid deformable registration proceeds as follows. To isolate the motion of each rigid bony structure, a subvolume is defined in the CT data using the bounding boxes of each segmented vertebra. These (possibly overlapping) subvolumes are then independently aligned to the MR data again using rigid transformations determined by the mutual information cost function and Powell's optimization method.
In this case, the CT voxels are masked so that only voxels within the boundaries of the bony tissue contribute to the cost function. For N separate vertebrae, the local registrations result in $\mathrm{N}$ separate rigid transformations.

At this point, a description is available that properly aligns those parts of the MRI and CT data representing bony tissue, but not for the adjacent soft tissue. To align the soft tissue, a smoothly varying deformation is computed using an approximating TPS algorithm [7], [8]. The TPS technique requires a sparse set of landmarks that are to be brought into correspondence. These landmarks are automatically obtained from the segmented data using simple edge-detection techniques and random sampling from the edge candidates. In $\mathbb{R}^{3}$ using derivatives of degree two, the desired basis functions are of the form $|\mathbf{r}|$, where $\mathbf{r}$ is the distance between the point being calculated and a landmark point. The TPS formulation also includes a smoothness parameter, which changes the spline from interpolation (i.e. the precise matching of landmark points between images, which occurs when the parameter is equal to zero) to approximating, which still attempts to bring the landmark points into correspondence but now also takes into consideration a smoothness constraint. This approximation parameter provides a characterization of the stiffness of the soft tissue. Automatic estimation of an appropriate stiffness factor is obtained iteratively by using the mutual information cost function on the result of the overall hybrid deformable transformation. Due to the complex response of the cost function to parameter changes, including many local optima, we have simplified the optimization problem by seeking the local maximum closest to the parameter value zero. This simplification is based on the assumption that the soft tissue must be flexible enough to allow bone movement, so the tendency should be toward landmark correspondence rather than smoothness. The resulting parameter vs. cost function curves computed during this project support this assumption, but more work is needed to see if it holds true in general. The iterative procedure first seeks an interval containing this local maximum then employs Brent's method to find the optimum.

\section{Results}

As a demonstration of the algorithm, it is first applied to a simplified dataset showing exaggerated motion, seen in Fig. 1. The L2 and L4 segmented vertebrae from the volunteer dataset were isolated to form a simplified reference phantom, as seen in the top row of Fig. 1. Each vertebra was separately rotated and translated to produce a deformed dataset simulating patient movement, shown in the middle row. The registration algorithm was then applied to the deformed volume to arrive at a registered volume, seen on the bottom row. Grid lines have been added to the registered data to show the transformation. It is seen that the grid lines remain orthogonal within the vertebrae of the registered volume, indicating a rigid local transformation in those regions. The surrounding soft tissue, however, was allowed to deform smoothly according to the TPS. 
Results on the cadaver spine images are seen in Fig. 2. An edge map of the approximate vertebral surface boundaries from the reference CT image is superimposed on the target MR images to better compare their alignment. This dataset has a large range of motion so we can see the improvement given by the subvolume local registration in an actual image as opposed to just in the simple phantom. However, much of the soft tissue that is present in living beings has been stripped from the cadaver spine so the improvement from the non-rigid portion of the hybrid registration is not as clear as it might be in clinical images.

Next we consider the results from the volunteer dataset to test the algorithm on a more clinically relevant and realistic set of data. Results on the volunteer dataset are shown in Fig. 3. Again, edge maps from the reference CT are shown for each step of the algorithm. The first two rows show the original $\mathrm{CT}$ and MR datasets. A globally rigid registration obtained using the mutual information-based technique brings the datasets into fairly close alignment. Finally, the hybrid technique using the individually computed rigid transformations along with the TPS is seen on the bottom. Because in this case, the non-rigid component of the motion between the CT and MRI data was fairly small, it is hard to appreciate the difference in registration quality between the rigid and hybrid approaches. However, the mutual information between the CT and the TPS version is greater than for the rigidly aligned version, indicating that the TPS registration is more accurate. In this case, the improvement in mutual information between the hybrid and global registrations is about $6 \%$ of the improvement between the original and the globally registered version.

Future work will investigate the effect segmentation quality has on the resulting registration. Since the rigid/nonrigid boundary and the location of the control points are determined based on the edges of the segmentation, the accuracy of the registration could depend greatly on the accuracy of the segmentation. In addition, we will test the effects of increasing or decreasing the number of control points, especially since this number has a large effect on computation time. Changing the criteria for selecting control points from the segmented edge candidates may also affect the registration accuracy. Finally, we will include the registration of $\left[{ }^{18} \mathrm{~F}\right]$ fluoride ion PET bone images and evaluate the algorithm performance on this different modality.

\section{ACKNOWLEDGMENT}

The authors would like to thank Dr. Thomas Budinger for his ideas on the motivation for this work, Julio Carballido-Gamio for his segmentation of the cadaver dataset, and Scott Yerby for the MRI cadaver dataset and use of the cadaver spines.

This work was supported by the National Institute on Aging, U.S. Department of Health and Human Services under grant R01-AG17762, by the Director, Office of Science, Office of Biological and Environmental Research, Medical Sciences Division of the U.S. Department of Energy under contract DE-AC03-76SF00098, and by a National Science Foundation Graduate Research Fellowship.

\section{REFERENCES}

[1] A. Panigrahy, S. D. Caruthers, J. Krejza, P. D.Barnes, S. G. Faddoul, L. A. Sleeper, and E. R. Methem. Registration of threedimensional MR and CT studies of the cervical spine. Am J Neuroradiol, vol. 21, pp. 282-289, 2000.

[2] Y. C. Tai, K. P. Lin, C. K. Hoh, S. C. H. Huang, and E. J. Hoffman. Utilization of $3 \mathrm{D}$ elastic transformation in the registration of chest X-ray CT and whole body PET. IEEE Trans Nucl Sci, vol. 44, no. 4, pp. 1606-1612, 1997.

[3] J. A. Little, D. L. G. Hill, and D. J. Hawkes. Deformations incorporating rigid structures. Comput Vision Image Understand, vol. 66 , no. 2, pp. 223-232, 1997.

[4] F. Maes, A. Collignon, D. Vandermeulen, G. Marchal, and P. Suetens. Multimodality image registration by maximization of mutual information. IEEE Trans Med Imaging, vol. 16, no. 2, pp. 187-198, 1997.

[5] J. P. W. Pluim, J. B. A. Maintz, and M. A. Viergever. Image registration by maximization of combined mutual information and gradient information. IEEE Trans Med Imaging, vol. 19, no. 8, pp. 809-814, 2000.

[6] J. P. W. Pluim, J. B. A. Maintz, and M. A. Viergever. Mutual information matching in multiresolution contexts. Image Vision Comput, vol. 19, pp. 45-52, 2001.

[7] F. L. Bookstein. Principal warps: Thin-plate splines and the decomposition of deformations. IEEE Trans Pattern Anal Mach Intell, vol. 11, no. 6, pp. 567-585, 1989.

[8] K. Rohr, H. S. Stiehl, R. Sprengel, T. M. Buzug, J. Weese, and M. H. Kuhn. Landmark-based elastic registration using approximating thin-plate splines. IEEE Trans Med Imaging, vol. 20, no. 6 , pp. 526-534, 2001. 

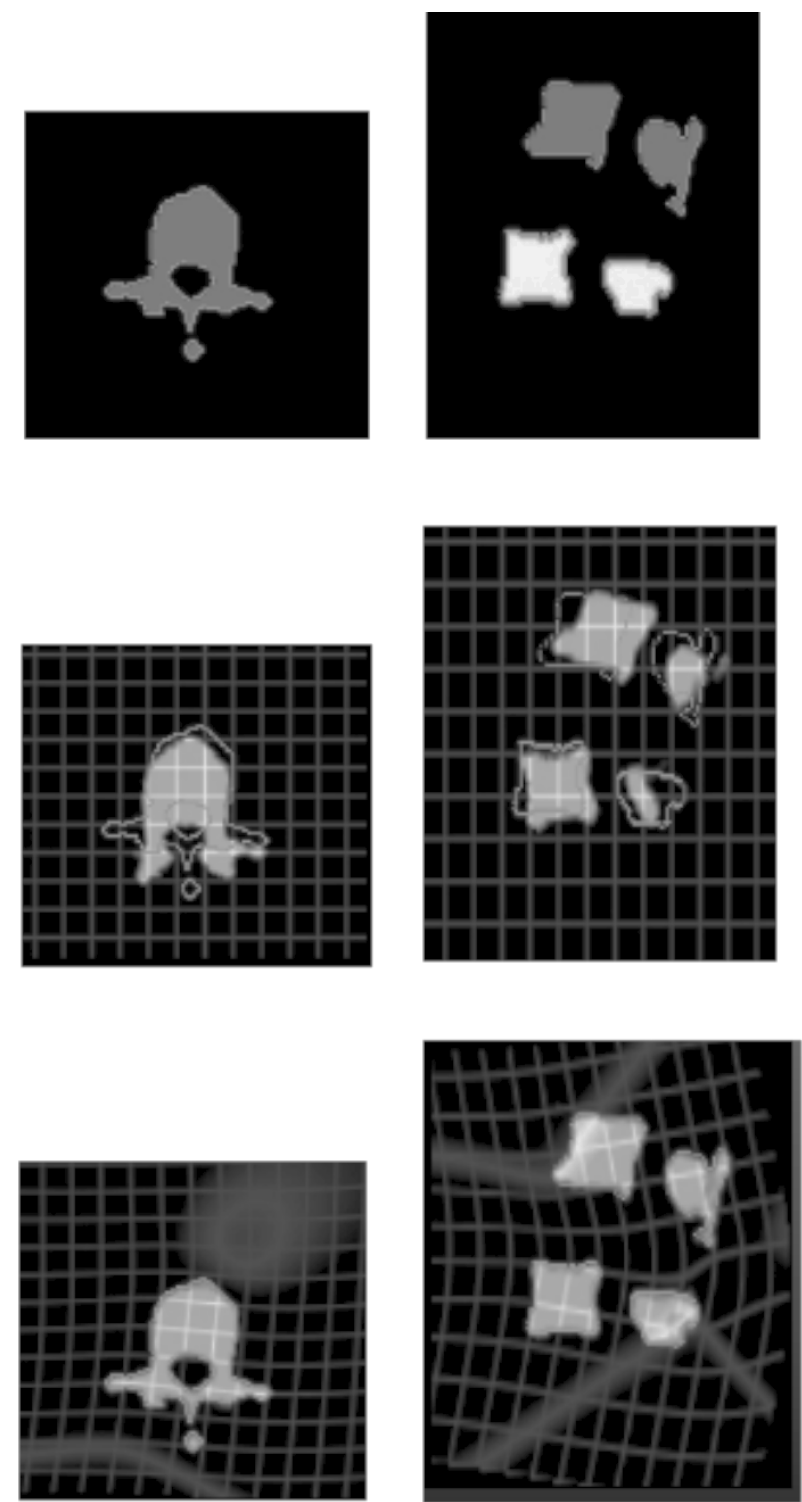

Fig. 1. Simplified dataset with large motion. Axial and sagittal views of the reference data (top) are shown above a deformed version, or target volume (middle) that is to be re-registered with the reference. The registered version is seen on the bottom. Grid lines placed on the target volume and deformed during the registration show that the segmented vertebrae are rigidly displaced, yet the background varies smoothly.
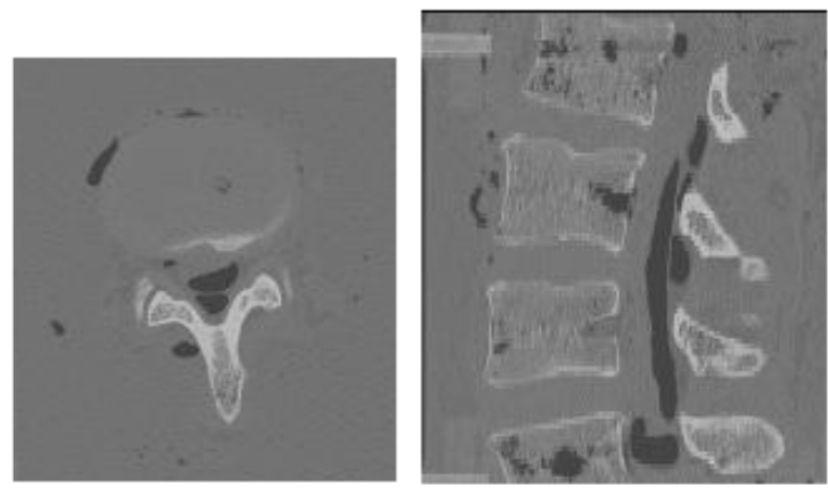

(a) Reference CT
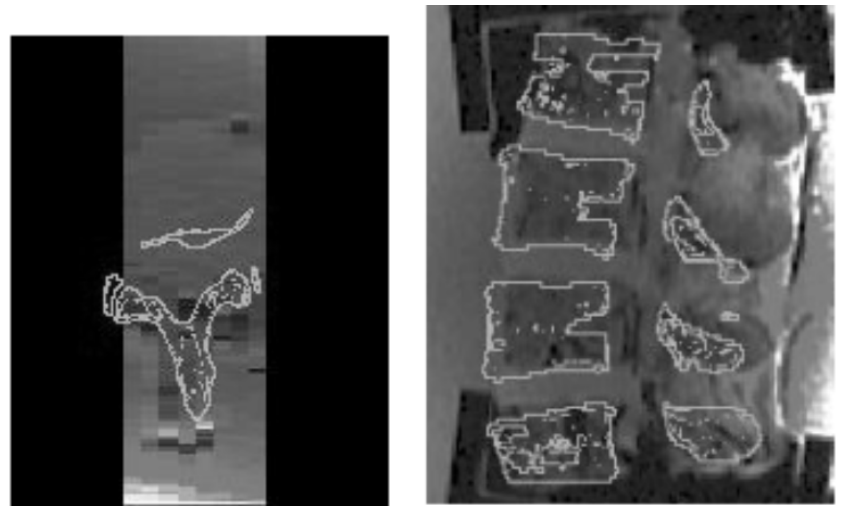

(b) MRI after global registration

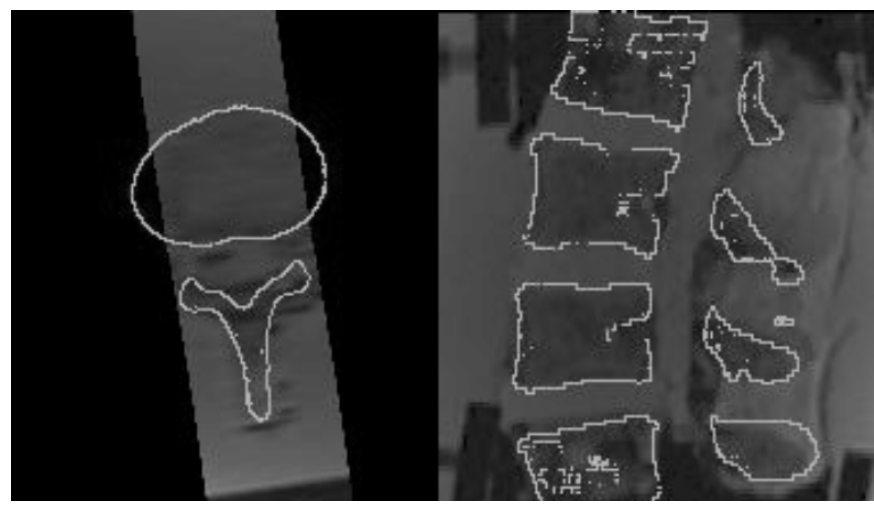

(c) MRI after hybrid registration

Fig. 2. Results on image volumes of a cadaver lumbar spine, axial and sagittal views. The reference CT data were acquired with the cadaver spine in a neutral position, and the MRI was taken with the spine in flexion. Edge maps of approximate vertebral boundaries from the reference CT image are shown on the target MR images to assist in viewing the alignment. 


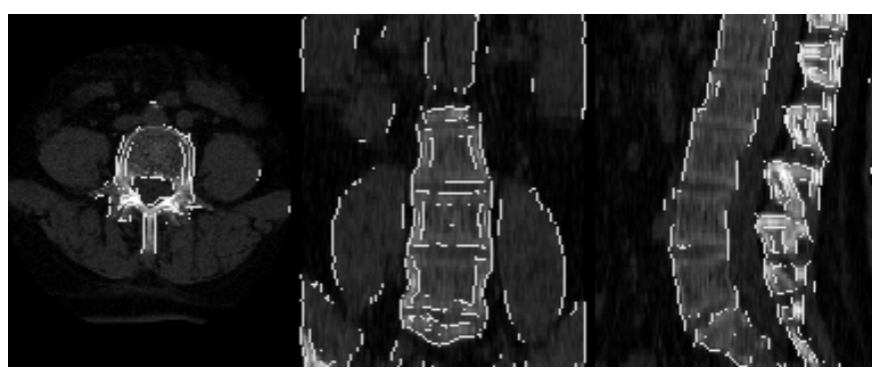

(a) Reference CT image

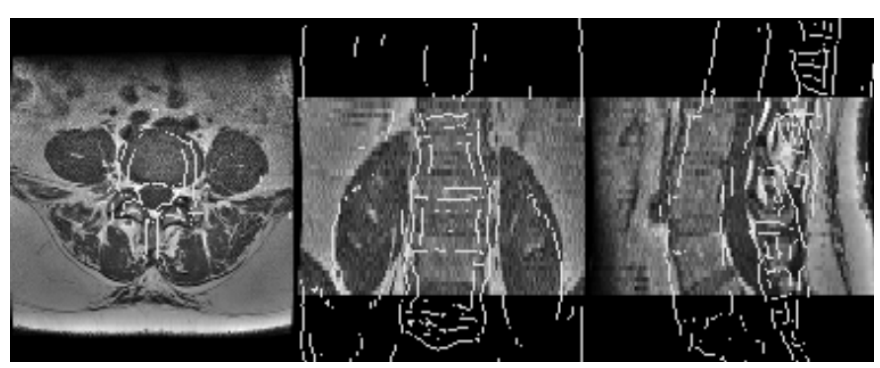

(b) Original MR image

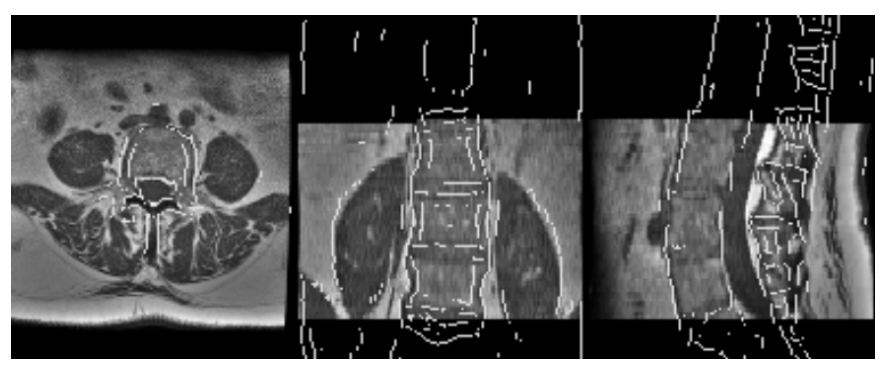

(c) Globally registered MR image

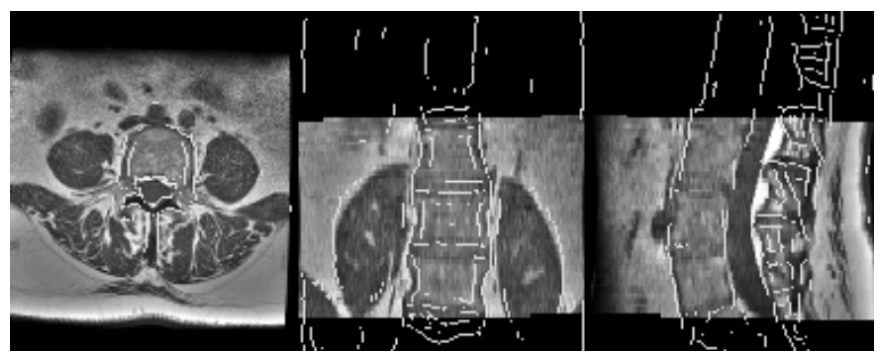

(d) Hybrid registration of MR image

Fig. 3. Results on CT and MR images of a volunteer. Axial, coronal, and sagittal views. Edge maps from the reference data help show alignment. 